\title{
A High Transmission Hemispherical Energy Analyzer for Ion Spectrometry
}

\author{
Stephen G. Anderson, Karl W. Sohlberg, Stephen L. Howard, \\ Anil K. Shukla, and Jean H. Futrell \\ Department of Chemistry and Biochemistry, University of Delaware, Newark, Delaware, USA
}

\begin{abstract}
A hemispherical energy analyzer was constructed by using a novel approach to control the fringing electrostatic field. It provides several properties useful in ion spectrometers: namely, rather simple fabrication and compact size, high transmission efficiency at moderate resolution, and the capability to adjust resolution by changing the intersphere polentials. A computer program was developed to evaluate ion trajectories through the hemispherical analyzer. Data obtained from the trajectories were used to predict the characteristics of the analyzer. Expcriments performed to determine the kinetic energy dependence of the absolute transmission and the resolution functions are in accord with theoretical calculations. (J Am Soc Mass Spectrom 1991, 2, 506-510)
\end{abstract}

$\mathrm{K}$ inetic energy analysis of ions provides uniquely valuable information for ion collision dynamics studies. This analysis is usually accomplished by one of two means-measurement of the time of flight of an ion over a known distance or measurement of the force required to produce a known deflection in the ion trajectory while passing the ions through well-defined magnetic or electric fields. The former method is appropriate for puised experiments whereas the latter is suitable for both continuous and pulsed experiments. Electrostatic analyzers commonly used include retarding field analyzers, mirror analyzers, and various dispersive analyzers. Dispersive analyzers that employ cylindrical or hemispherical plates can readily achieve energy resolution of a few tens of millielectronvolts. Hemispherical analyzers have an added advantage of compactness. These analyzers use an electric field proportional to $1 / r^{2}$ produced by placing a potential across a pair of concentric hemispheres. This electric field also produces focusing in both the deflection plane and the plane perpendicular to deflection.

The performance of electric field analyzers is frequently limited by the technique used to terminate the dispersing freld at the boundaries of the device. Fringing fields at the entrance and exit of the analyzer cause undesirable aberrations that distort ion trajecto ries and both degrade the resolution of the analyzer and change the physical characteristics (i.e., spot size and divergence angles) of the ion bcam. Fringing fields effects become more intractable as the gap between the plates of the analyzer increases.

Address reprint requests to Jean H. Futrell, Department of Chemistry and Biochemistry, University of Delaware, Newark, DE 19716.
For hemispherical analyzers, Herzog [1] and Wollnik [2] have described field shunts which overcome this problem by applying potentials on these shunts identical to the centerline equipotential of the analyzer. In this scheme the hemispheres are truncated at some angle $\gamma$, and the shunts are placed at such a distance from the entrance and exit apertures that a "hemispherical" analyzer is created in which only the central $180-2 \gamma$ degree sector is contained between the electrodes. Nishigaki and Kanai [3] have performed numerical calculations to determine the sector angle that produces optimum focusing. Unfortunately, difficulty in fabrication and mounting of truncated hemispheres with a wide gap (chosen for high transmission and variable effective energy resolution) makes this solution inappropriate for our application, in which the energy width for product ions varies from $<1 \mathrm{eV}$ near threshold to $>100 \mathrm{eV}$ at kilovolt energies.

We have recently constructed a crossed-beam tandem hybrid mass spectrometer with a supersonic neutral beam system to study the dynamics of collisioninduced dissociation (CID) processes [4]. This instrument was designed to study CID processes over a broad range of laboratory collision energies (viz., 1-3000 eV) and employs a rotatable analyzer to investigate the angular dependence of daughter ion products. Kinetic energy analysis of fragment ions is essential to determine the energetics and to characterize the operative mechanisms of CID processes.

In the CID process, kinetic energy released on dissociation is amplified in the laboratory frame [5], resulting in broad energy distributions typically of the order of several electronvolts. Except at low energy, our application requires only modest energy resolu- 
tion. However, the use of a supersonic beam as a source of neutrals instead of a collision cell and the sampling of a cone of ions scattered over a range of angles after collision imposes a requirement of very high transmission efficiency. The preferred solution is a variable resolution device which ranges from -0.1 $\mathrm{eV}$ at low energy to a few electronvolts for high energy experiments. A $180^{\circ}$ hemispherical energy analyzer with large apertures and a wide gap between the hemispheres fulfills all the above requirements with the additional advantage of compact geometry.

Siegel and Vasile [6] have recently described an energy analyzer/quadrupole mass filter combination for use in static secondary-ion mass spectrometry work. Their device is a $180^{\circ}$ radial field electrostatic deflector analyzer with large apertures but with no outer hemisphere (the vacuum housing at ground potential actually serves as the outer hemisphere). A conically tapered resistive disk with a single conducting (inner) hemisphere produces the required $1 / r^{2}$ electric field and establishes boundary conditions at the entrance and exit apertures. Although this SphericEL device (Extrel Corporation, Pittsburgh, PA) is an excellent choice for many applications, a commercially fabricated larger scale disk constructed for us by Extrel Corporation exhibited resistance changes in the disk with use. This deterioration in performance while the prototype device was being tested led us to seek an alternative solution.

One consequence of a large gap between the hemispheres is that the fringing fields at the boundary of the analyzer extend a significant distance beyond the geometric boundaries of the device. If uncorrected these fringing fields would result in an unacceptable distortion of ion trajectories. Figure 1 illustrates both the analyzer and our novel solution for terminating the electric field sharply at the geometric boundaries of the device. The relatively simple procedures for constructing such a device are described and experi- mental confirmation of predicted performance characteristics is presented.

\section{Analyzer Description and Operation}

The analyzer shown schematically in Figure 1 is constructed with two concentric stainless steel hemispheres with a common geometric center. The inner and outer hemisphere radii are 2.9 and $7.6 \mathrm{~cm}$, respectively. The surfaces of the hemispheres exposed to ions are painted with colloidal graphite to minimize surface charge buildup and reflection of ions. The hemispheres are bolted to a double-faced fiberglass electronics printed circuit board (pc-board). The pcboard between the two hemispheres is etched with 10 solid concentric rings which define and terminate the radial field at the boundaries. The density of rings is highest near the exit and entrance apertures and is lowest next to the hemispheres where field penetration has minimum effect. Entrance and exit apertures of 0.8 - and 1.6-mm diameter, respectively, cut through the pc-board are symmetrically placed at a radius of $3.8 \mathrm{~cm}$. The rings are electrically connected between the hemispheres by a voltage divider chain of metal foil resistors of appropriate values to establish a $1 / r^{2}$ electric field when a potential difference is applied across the hemispheres. The total resistance of the divider chain is established by considerations of electrical RC time constants (since the device is designed for both static and scanning operation) and power supply loading. For our applications a total resistance of $500 \mathrm{k} \Omega$ was selected so that a voltage difference as large as $300 \mathrm{~V}$ across the two hemispheres could be applied. This choice allowed us to cover the broad range of ion kinetic energies observed experimentally in CID studies.

The radial potential in the hemispherical analyzer is established by the voltage difference between the

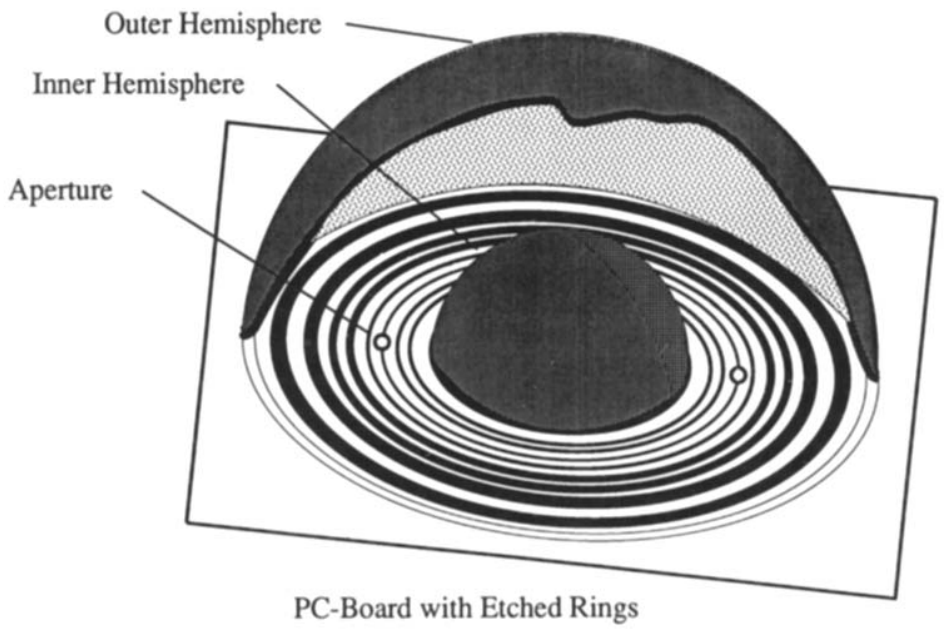

Figure 1. A perspective view of the hemispherical energy analyzer showing inner and outer hemispheres attached to a printed circuit board with copper conducting rings etched between the two hemispheres. 
two spheres which define the field gradient. The kinetic energy of a single jon through the analyzer is given by

$$
W_{0}=\frac{1}{2} \frac{V_{2}-V_{1}}{R_{1}}-\frac{1}{R_{2}} \times \frac{1}{R_{0}}=k_{p}\left(V_{2}-V_{1}\right)
$$

where $W_{0}$ is the kinetic energy of the ion, $R_{0}$ is the radial spacing of the entrance and exit apertures (assumed to be symmetrical), $R_{1}$ and $R_{2}$ are the radii of inner and outer hemispheres, and $V_{1}$ and $V_{2}$ are voltages applied to inner and outer hemispheres, respectively [6, 7]. The transmitted energy is proportional (with proportionality constant $k_{p}$ ) to the potential difference between the hemispheres. Therefore, the energy spectrum can be obtained by scanning either the potential difference or the accelerating potential.

To terminate the fringing fields at the entrance aperture, the potential on the entrance aperture must be matched to the ion energy. This potential is determined by solving for the potential of the last lens element $V_{L}$ at the centerline radius of the aperture, $R_{0}$,

$$
V_{L}=\frac{\left(\frac{V_{2}}{R_{1}}-\frac{V_{1}}{R_{2}}\right)+\left(V_{1}-V_{2}\right)}{\frac{1}{R_{1}}-\frac{1}{R_{2}}} \cdot \frac{1}{R_{0}}
$$

The energy resolution is related to the size of the entrance and exit apertures and the centerline radius $R_{0}$. If the entrance aperture location is defined by $R_{0}+\Delta r_{i}$ and the exit aperture by $R_{0}+\Delta r_{f}$, the resolution for the simplest class of planar trajectories entering normal to the entrance aperture (circular orbits) is given by

$$
\frac{\Delta W}{W_{0}}=\frac{1}{2 R_{0}}\left[\Delta r_{i}+\Delta r_{f}\right]
$$

where $\Delta r_{i}$ and $\Delta r_{f}$ are half of the respective aperture diameters [6]. If one aperture is much larger, the resolution is related to this resolution-determining aperture as

$$
\frac{\Delta W}{W_{0}}=\frac{\Delta r}{R_{0}}
$$

where $\Delta r$ is half of the larger aperture diameter. If the analyzer is coupled with other detector elements, this resolution function is then convoluted with the resolution functions of those detector elements to compute an overall resolution function. (It should be noted that the symmetry of the radial analyzer allows us to treat all trajectories as if they occurred in one plane because there is no angular dependence of the $1 / r^{2}$ field. Elliptical trajectories require modeling calculations for evaluation of transmission and resolution, as discussed by Siegel and Vasile [6], and do not lead to a simple analytical expression.)

A common practice for measuring ion energy distributions by using electrostatic analyzers is to scan potential on one sphere while keeping the other constant. In this method, the centerline potential also changes continuously and more lens elements are often required to maintain the correct potential at the entrance. A more convenient way to scan the analyzer is to maintain fixed potential across the hemispheres while scanning the centerline potential. This procedure maintains constant resolution for the scan. This can be readily achieved by using an external resistor bridge of approximately an order of magnitude less resistance connected in parallel to the resistor bridge between the hemispheres. This allows the centerline potential, $V_{0}$, to be varied independently of the potential, $V_{p}$, across the hemispheres. The potentials on the hemispheres and the scanning voltage circuit are depicted in Figure 2. In this manner, the correct field gradient is established for any potentials, $V_{1}$ and $V_{2}$, applied to the inner and outer hemispheres, respectively [6].

A test ion source was fabricated from a stainless steel block with high transmission grids welded across the electron entrance aperture to minimize field penetration that would otherwise perturb the ion energy distribution. The ion source performance characteristics were first measured by using a high resolution double-hemispherical energy analyzer of conventional design. These measurements determined that the source generated a Gaussian distribution of $\mathrm{Ar}^{+}$with

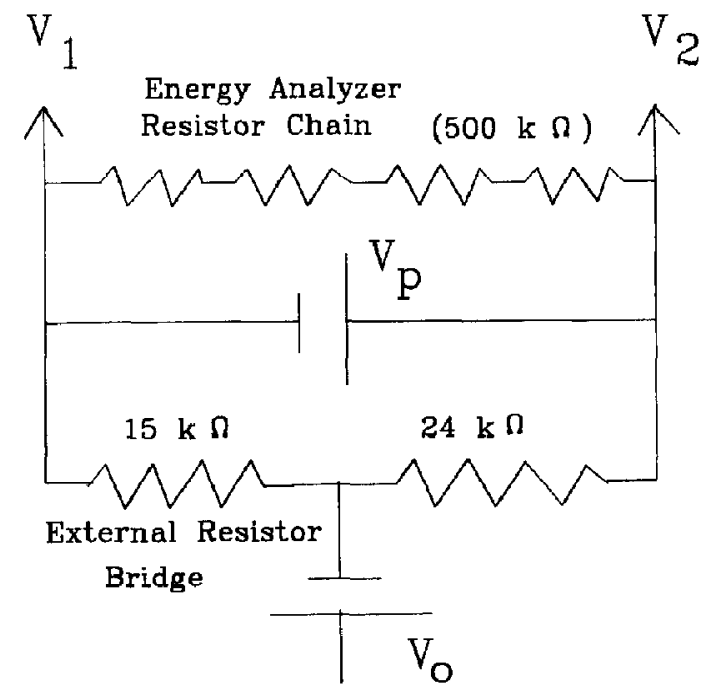

Figure 2. Schematic diagram of the circuit used to provide potentials $V_{1}$ and $V_{2}$ to the inner and outer hemispheres, respectively, as functions of the voltage difference between the hemispheres, $V_{p}$, and the centeriine potential, $V_{0}$. This arrangement allows $V_{0}$ to be varied independently of $V_{p}$. 
a full-width at half-maximum (FWHM) width of $1.3 \pm$ $0.1 \mathrm{eV}$ when operated with the same parameters used in the present experiment.

This source was mounted behind the entrance aperture and an electron multiplier was mounted opposite the exit aperture to measure transmitted ion current. The amplified current from the multiplier was measured with an electrometer (model 600B, Keithley, Inc., Taunton, MA). To determine the ion current entering the analyzer, a large diameter $(\sim 1.5$ $\mathrm{cm})$ aperture was drilled in the outer hemisphere directly behind the entrance aperture. The aperture and the entrance to the electron multiplier were covered with high transmission grids to prevent distortion of the outer field boundary. The ion current entering the energy analyzer was then monitored by setting the potential across the hemispheres to zero volts so that ions would continue on straight line trajectories from the entrance aperture through the large aperture in the outer hemisphere. The ions were collected on a Faraday plate placed behind the outer hemisphere. Ion transmission was defined as the ratio of the current detected after the final exit aperture to the current at the Faraday plate.

\section{Results and Discussion}

\section{Transmission Characteristics}

A computer program was developed for the Macintosh Plus computer to calculate ion trajectories through the analyzer [6-8] and to predict the transmission of this analyzer at a given resolution. The program traces ion trajectories on the graphics display after all the input parameters are given. Upon successful completion of a trajectory, the user is informed whether the trajectory was "successful" (passes through the final aperture) and a new trajectory is started. The program was used to determine the analyzer transmission function for a given set of analyzer operating parameters.

At $90-\mathrm{eV}$ transmission energy, the transmission function for 0.8- and $1.6-\mathrm{mm}$ diameter apertures is graphically depicted in Figure 3 by the dashed curve. This figure also shows an experimental energy spectrum (solid curve) at $90 \mathrm{eV}$ as measured by the analyzer and described below. The experimental profile is the convolution of the energy distribution from the source with the transmission function shown in Figure 3. The width of the apparatus function, which is trapezoidal in shape, is $1.1 \mathrm{eV}$ FWHM. The experimental curve is broader than the calculated function, and the combined FWHM is $3.3 \mathrm{eV}$.

Taking the potential difference between the source block and the foil backing of the pc-board (also electrically connected to the centerline ring) as the nominal energy of the ions, the absolute transmission is plotted in Figure 4 as a function of transmission energy. This graph shows the absolute transmission of the

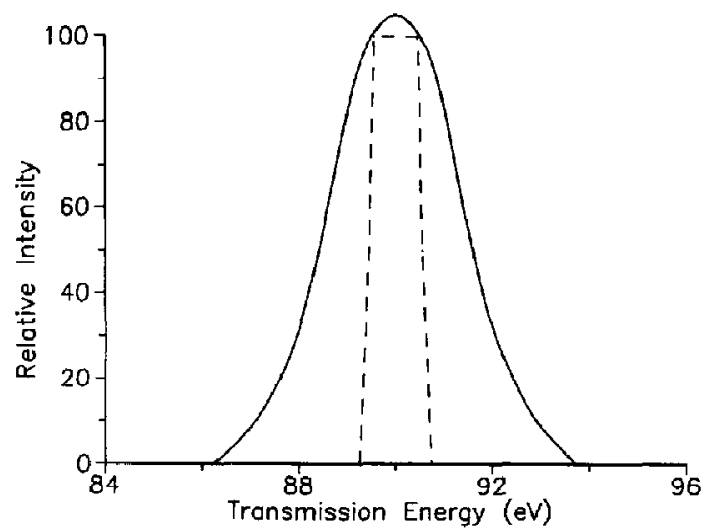

Figure 3. Transmission function of energy analyzer (dashed curve) at $90-\mathrm{eV}$ transmission energy. This function is constructed with an experimental energy distribution of $\mathrm{Ar}^{+}$measured at the same transmission energy.

analyzer is essentially constant for ion energies above $30 \mathrm{eV}$.

\section{Energy Analysis}

The FWHM peak widths were determined as a function of transmission energy. These data are plotted as points in Figure 5 with the predicted peak widths (FWHM) calculated from eq 4 shown as a solid line. A least-squares fit of the data is shown by a dashed line. This line fits the equation $\Delta W=0.022( \pm 0.001) W+$ $1.30( \pm 0.03)$. The offset of $1.30( \pm 0.03) \mathrm{eV}$ is in excellent agreement with the calibrated $1.30-\mathrm{eV}$ peak width (FWHM) of the ion beam provided by the ion source. The theoretical slope of the line calculated from eq 4 is 0.021 compared with the experimental slope of 0.022 . These data demonstrate that peak width is only slightly affected by instrument functions. Considering the wide gap between the hemispheres and the simplistic solution for controlling the field boundary, the agreement between theory and experiment is remarkably good.

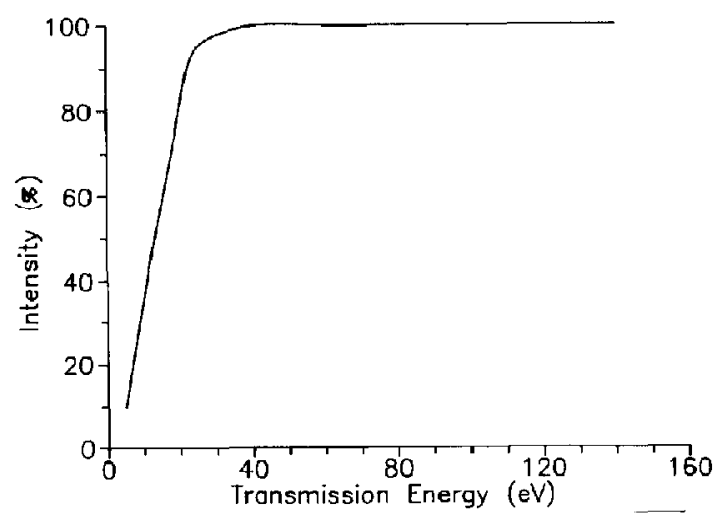

Figure 4. Absolute transmission of the energy analyzer as a function of nominal transmission energy. 


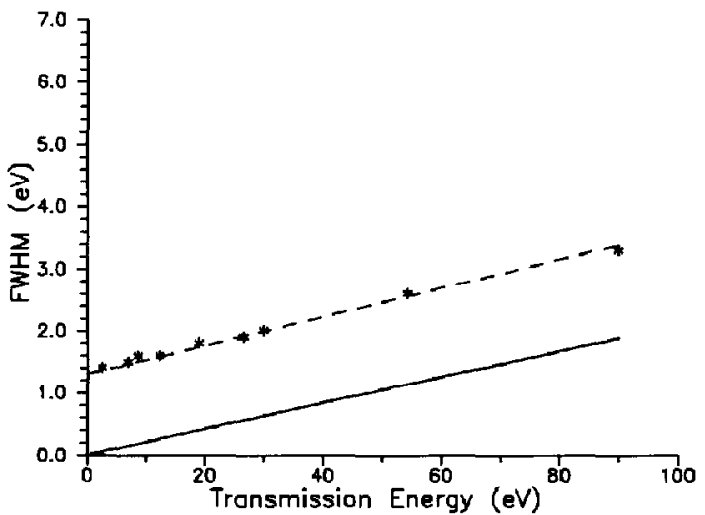

Figure 5. FWHM of the transmitted beam as a function of transmission energy with $0.8-\mathrm{mm}$ diameter entrance aperture and $1.6-\mathrm{mm}$ diameter exit aperture. Solid line indicates the theoretical values from eq 4 for this analyzer. Experimental values plotted in the dashed line were obtained with an $\mathrm{Ar}^{+}$ beam from an ion source with a nominal FWHM of $1.30 \mathrm{eV}$.

Figure 6 shows the correlation of experimental $V_{p}$ and $V_{0}$, which produces the maximum transmission of the ion beam. The slope $(0.63 \pm 0.02)$ of the leastsquares averaged line is in very gond agreement with the value of 0.617 predicted by eq 1 . This further demonstrates that either $V_{p}$ or $V_{0}$ can be scanned to obtain an energy spectrum. The line also shows that absolute ion energy can be measured with an accuracy of better than $2 \%$ at higher energies.

To test for effects of field penetration at the apertures, we enlarged the apertures by a factor of two in a second prototype energy analyzer. Spectra were taken and the FWHM of the resulting profiles were plotted in Figure 7 . The experimental data have a slope of 0.058 compared to theoretical slope of 0.042 obtained from eq 4 . The agreement between theory and experiment is decreased in comparison with Fig-

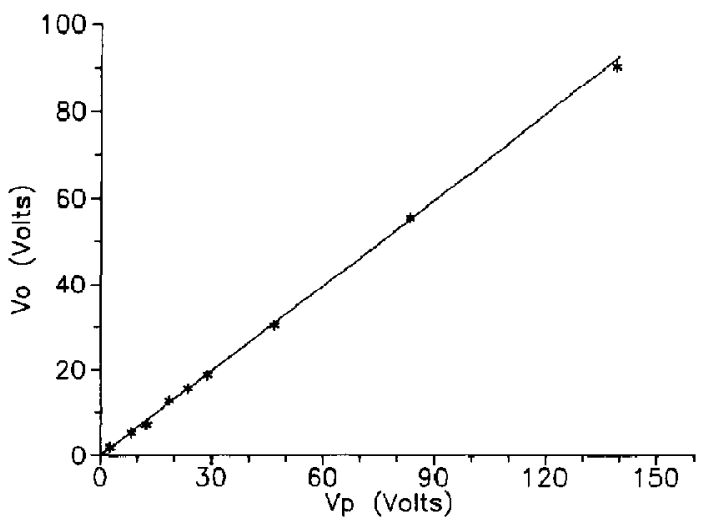

Figure 6. Plot of the centerline potential $\left(V_{0}\right)$ versus potential difference $\left(V_{p}\right)$ between the hemispheres for the maximum transmission of the ion beam.

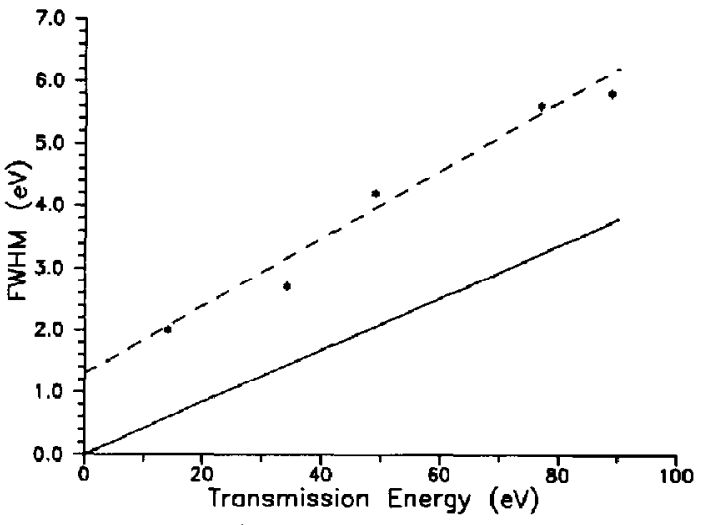

Figure 7. FWHM of the transmitted beam as a function of transmission energy with $1.6-\mathrm{mm}$ diameter entrance aperture and $3.2-\mathrm{mm}$ diameter exit aperture.

ure 5 and shows the anticipated effect of field penetration. The intercepts of the two curves are again offset by $1.30 \mathrm{eV}$, the independently measured width of the ion distribution obtained from the ion source. Increasing the apertures by a factor of two doubles the slope theoretically (decreasing the resolution by the same factor) while the experimental slope changed by a factor of 2.6. With the enlarged apertures ion transmission is excellent at $20 \mathrm{eV}$ (comparable to $40 \mathrm{eV}$ in the Figure 4 analyzer), at which point the resolution is $-1 \mathrm{eV}$ (Figure 7). This is entirely adequate for CID experiments. For other applications smaller apertures can be used with typical energy resolution of several tenths of an electronvolt.

\section{Acknowledgments}

The authors wish to express their appreciation to Professor Douglas P. Ridge, Dr. A. J. H. Boerboom, and Dr. Alan L. Rockwood for many fruitful and stimulating discussions on the theory and construction of the analyzer. We are also most grateful for the support of this work by National Science Foundation grant $\mathrm{CHE}-8312069$.

\section{References}

1. Herzog, R. Z. Phys. 1940, 47, 18.

2. Wollnik, H. Optics of Charged Particles; Academic: Orlando, FL, 1987.

3. Nishigaki, S.; Kanai, S. Rev. Sci. Instrum. 1986, 57, 225.

4. Shukla, A. K.; Anderson, S. G.; Howard, S. L.; Sohlberg, K. W.; Futrell, J. H. Int. J. Mass Spectrom. Ion Processes 1989, 92, 147.

5. Cooks, R. G.; Beynon, J. H.; Caprioli, R. M.; Lester, G. R. Metastable Ions; Elsevier: New York, 1973, pp 57-85.

6. Siegel, M. W.; Vasile, W. J. Rev. Sci. Instrum. 1981, 52, 1603.

7. Becker, R. In Elect romagnetic Fields and Interactions; Sauter, F., Ed.; Dover: New York, 1982

8. Goldstein, H. Classical Mechanics; Addison-Wesley: Reading, MA, 1950. 